\title{
NEK7 Coordinates Rapid Neuroinflammation After Subarachnoid Hemorrhage in Mice
}

\author{
Gen Li ${ }^{1,2+}$, Yushu Dong ${ }^{1+}$, Dongdong Liu ${ }^{1,2}$, Zheng Zou ${ }^{1}$, Guangzhi Hao ${ }^{1}$, Xu Gao ${ }^{1}$, \\ Pengyu Pan ${ }^{1 *}$ and Guobiao Liang ${ }^{1 *}$ \\ ${ }^{1}$ Department of Neurosurgery, General Hospital of Northern Theater Command (General Hospital of Shenyang Military \\ Command), Shenyang, China, ${ }^{2}$ Dalian Medical University, Dalian, China
}

OPEN ACCESS

Edited by:

Emmanuel Pinteaux, University of Manchester,

United Kingdom

Reviewed by:

Maria Calvo-Rodriguez, Harvard Medical School,

United States

Paul Kasher,

University of Manchester,

United Kingdom

*Correspondence:

Pengyu Pan

panpengyu09@sina.com

Guobiao Liang

liangguobiao6708@163.com

tThese authors have contributed equally to this work

Specialty section:

This article was submitted to

Stroke,

a section of the journa

Frontiers in Neurology

Received: 02 June 2019

Accepted: 15 May 2020

Published: 08 July 2020

Citation:

Li G, Dong Y, Liu D, Zou Z, Hao G,

Gao X, Pan P and Liang G (2020)

NEKT Coordinates Rapid

Neuroinflammation After

Subarachnoid Hemorrhage in Mice.

Front. Neurol. 11:551.

doi: 10.3389/fneur.2020.00551
Background: Subarachnoid hemorrhage $(\mathrm{SAH})$ is a devastating disease which leads to high morbidity and mortality. Recent studies have indicated that, never in mitosis gene A-related expressed kinase 7 (NEK7), is involved in NLRP3 (NLR family, pyrin domain containing 3) associated inflammation, which may result in subsequent cellular and vascular damage. The aim of this study was to investigate whether NEK7 is involved in the pathophysiology of subarachnoid hemorrhage.

Methods: 455 adult male C57B6J mice, weighing 22 to $30 \mathrm{~g}$, were used to investigate the time course of NEK7 expression in the ipsilateral cortex after SAH, and to investigate the intrinsic function and mechanism of NEK7. A vascular puncture model was used to create the mouse SAH model, and intracerebroventricular injection was used to deliver NEK7 recombinant protein, NEK7 small interfering RNA, nigericin, and MCC950. Neurological score, brain water content, Evans blue extravasation, immunofluorescence, and western blot were evaluated for neurological outcome, neuronal apoptosis, blood-brain barrier damage, microglia accumulation, and the mechanism of NEK7 and NLRP3 activation.

Results: Our results exhibited that intrinsic NEK7 was elevated after SAH in the cortex of the left/ipsilateral hemisphere and was colocalized with microglia, endothelial cells, neuron, astrocyte, and oligodendrocyte, and highly expressed in microglia and endothelial cells after SAH. NEK7 recombinant protein aggravated neurological deficits, brain edema, neuronal apoptosis, BBB permeability, microglial accumulation, and activated caspase-1 and IL-1 $\beta$ maturation, while NEK7 small interfering RNA injection reversed those effects. Nigericin administration enhanced ASC oligomerization, caspase1 and IL-1 $\beta$ maturation without increasing the protein level of NLRP3, and ASC oligomerization and caspase-1 IL-1 $\beta$ maturation reduced when combined with NEK7 knockdown or MCC950 delivery. We found the level of NEK7 expression increased after $\mathrm{SAH}$ and could activate the downstream NLRP3 pathway to induce caspase- $1, \mathrm{IL}-1 \beta$ expression and then increased the BBB opening, microglia accumulation and neuronal apoptosis after SAH. 


\begin{abstract}
Conclusions: This study demonstrated for the first time that NEK7 mediated the harmful effects of neuronal apoptosis and BBB disruption after SAH, which may potentially be mediated by the NEK7/NLRP3 signal. NEK7 served as a co-component for NLRP3 inflammasome activation after SAH. NEK7 may be a promising target on the management of SAH patients.
\end{abstract}

Keywords: subarachnoid hemorrhage, microglia, NEK7, NLRP3, neuroinflammation, blood-brain barrier

\section{INTRODUCTION}

Despite years of efforts, early brain injury-the main contributor to the mortality and poor prognosis of patients after subarachnoid hemorrhage (SAH) - remains a catastrophic complication of a ruptured intracranial aneurysm (1). The pathophysiology of SAH involves an alteration in the brain neural vascular network, involving the arterial, capillary, venous systems, neurons, microglia, surrounding support cells, and the extracellular matrix (2). The precise mechanism of neuronal apoptosis and BBB damage, however, remains elusive.

The NLR family, pyrin domain containing 3 (NLRP3) inflammasome, consists of NLRP3, apoptosis-associated speck-like domain (ASC) and pro-caspase-1. It is a group of innate immune proteins considered to be sensors of pathogen- and damage- associated molecular patterns (3). NLRP3 inflammasome activation promotes the cleavage of pro-caspase 1 and pro- interleukin (IL)- $1 \beta$ into their mature forms, which contributes to the development of type 2 diabetes (4), atherosclerosis (5), gout (6), and Alzheimer's disease (7). Our previous study showed NLRP3 activation increased BBB extravasation, tight junction protein degradation, as well as neuronal apoptosis after CNS injury $(8,9)$. As a typical inflammation target, the exact mechanism of NLRP3 activation still remains unclear.

Recent studies reported that NEK7, a serine and threonine kinase involved in mitosis, is an essential activator of the NLRP3 inflammasome (10). NEK7-mutant cells showed lower caspase1 activation and IL-1 $\beta$ production in response to NLRP3activating stimuli (11). These data suggested that NEK7 acts as a switch between inflammasome activation and cell division (11). We postulated that NEK7 expression was altered after SAH and targeting NEK7 might inactivate NLRP3-inflammasome and downstream IL-1 $\beta$ to preserve neuronal death and $\mathrm{BBB}$ permeability. To elucidate the function of NEK7 after SAH, we analyzed the spatial distribution and time course of NEK expression, neurological function, neuronal apoptosis, and BBB permeability after NEK7 interference, and downstream NLRP3 activation.

\footnotetext{
Abbreviations: ANOVA, analysis of variance; ASC, apoptosis-associated specklike protein; BBB, blood-brain barrier; BAX, BCL-2-associated X protein; BCL-2, B-cell lymphoma 2; EBI, early brain injury; IL-1 $\beta$, Interleukin-1 beta; LRR, leucine rich repeat; NBD, nucleotide-binding domain; NEK7, Never in mitosis gene Arelated expressed kinase 7; NLRP3, NLR family, pyrin domain containing 3; PBS, phosphate-buffered saline; PYD, pyrin domain; SAH, subarachnoid hemorrhage; TJs, tight junctions; ZO-1, zonula occludens- 1 .
}

\section{MATERIALS AND METHODS}

\section{Animals}

All experimental procedures were approved by the Ethics Committee of the General Hospital of Norther Theater Command (Shenyang Military Command) and was performed in accordance with the guidelines in the National Institutes of Health Guide for the Care and Use of Laboratory Animals, and followed the ARRIVE guidelines. Four-hundred-and-fifty-five (455) adult male C57B6J mice (22 to $30 \mathrm{~g}$ ), were provided by the Department of Experimental Animals of the General Hospital of Norther Theater Command (Shenyang Military Command, Shenyang, China). Mice were acclimated in a reversed $12 \mathrm{~h}$ dark $/ 12 \mathrm{~h}$ light cycle environment and provided with free access to water and food.

\section{Experimental Design}

The present study contained three experiments, which were designed as follows.

\section{Experiment I}

To investigate the time course of NEK7 expression and cell type location of NEK7 after SAH, 44 mice were randomly assigned to seven groups: Sham $(n=6)$, SAH $3 \mathrm{~h}(n=6)$, SAH $6 \mathrm{~h}(n=6)$, SAH $12 \mathrm{~h}(n=6)$, SAH $24 \mathrm{~h}(n=6)$, SAH $48 \mathrm{~h}(n=6)$ and SAH $72 \mathrm{~h}(n=6)$. NEK7 protein expression was detected by Western blot in cortex isolated from the ipsilateral/left hemisphere. Immunohistochemical staining of NEK7, NeuN (neuron marker), GFAP (astrocyte marker), Lectin (endotheliocyte marker), Iba-1 (microglia marker), and NG2 (oligodendrocyte marker) was performed at $24 \mathrm{~h}$ post SAH induction to confirm the spatial distribution of NEK7 in the cortex $(n=6)$.

\section{Experiment II}

To define the intrinsic function of NEK7 and to screen for effective dosage of NEK7 recombinant protein after SAH, 162 mice were randomly assigned to eight groups: Sham $(n=24)$, SAH $(n=18)$, SAH+NS (normal saline, $2 \mu \mathrm{L})(n=24)$, $\mathrm{SAH}+\mathrm{NEK} 75 \mathrm{ng} / \mu \mathrm{L}(n=12), \mathrm{SAH}+\mathrm{NEK} 725 \mathrm{ng} / \mu \mathrm{L}(n=$ 12), SAH+NEK7 $100 \mathrm{ng} / \mu \mathrm{L}(n=24), \mathrm{SAH}+\mathrm{Scr}$ (scrambled) siRNA $(n=24)$, SAH+NEK7 siRNA $(n=24)$. Beam balance and modified Garcia tests were performed at 24 and $72 \mathrm{~h}$ after $\mathrm{SAH}$ to assess the neurological deficits in each group $(n=$ 6). Furthermore, brain water content was performed at 24 and $72 \mathrm{~h}$ post SAH. Evans blue extravasation assessment test at $24 \mathrm{~h}$ post $\mathrm{SAH}$ induction to evaluate the blood-brain barrier damage. Immunohistochemical staining was also performed to detect 
the neuronal apoptosis, endothelial continuity, and microglia accumulation in the cortex of ipsilateral/left hemisphere at $24 \mathrm{~h}$ after SAH induction $(n=6)$.

Furthermore, 30 mice were randomly divided into the following groups: Sham $(n=6), \mathrm{SAH}+\mathrm{NS}(n=6), \mathrm{SAH}+\mathrm{NEK} 7$ $100 \mathrm{ng} / \mu \mathrm{L}(n=6), \mathrm{SAH}+\mathrm{Scr}$ siRNA $(n=6), \mathrm{SAH}+\mathrm{NEK} 7 \mathrm{siRNA}$ $(n=6)$. Western blot was performed to detect the NEK7, NRLP3, ASC, caspase-1, IL-1 $\beta$, BCL-2, and BAX expression in cortex of the ipsilateral/left hemisphere in each group.

\section{Experiment III}

To validate the function of NEK7-denpdent NLRP3 signal activation after SAH induction, 84 mice were assigned to seven groups: Sham $(n=12)$, SAH $(n=12)$, SAH+Vehicle $(n=12)$, SAH+nigericin $(n=12), \operatorname{SAH}+$ nigericin+Scr siRNA $(n=12)$, $\mathrm{SAH}+$ nigericin+NEK7 siRNA $(n=12)$, and SAH+MCC950 $(n$ $=12$ ). Modified Garcia tests and beam balance were performed at $24 \mathrm{~h}$ post $\mathrm{SAH}$ to evaluate the neurological dysfunction and brain water content, and Evans blue extravasation assessments were carried out to test the blood-brain barrier permeability.

Furhermore, 72 mice were randomly divided into the following groups: Sham $(n=6)$, SAH+NS $(n=12)$, $\mathrm{SAH}+$ Ngericin $(n=12), \mathrm{SAH}+\mathrm{Ngericin}+\mathrm{Scr}$ siRNA $(n=12)$, SAH+Ngericin+NEK7 siRNA $(n=12)$, and SAH+MCC950 $(n=12)$. Immunohistochemical staining was also performed to detect the neuronal apoptosis, endothelial continuity, and microglia accumulation. Western blot was performed to detect the protein expression of NEK7, NLRP3, ASC, caspase-1, IL-1 $\beta$, BCL-2, and BAX.

\section{SAH Model}

SAH model was performed as previously described (12); mice were anesthetized with pentobarbital sodium $(40 \mathrm{mg} / \mathrm{kg})$ by intraperitoneal (i.p.) injection, then exposed the bifurcation of the common carotid artery in supine position. The left external carotid artery (ECA) was isolated and distally cut into a 2-mm stump. A 5-0 sharpened monofilament nylon suture was inserted into the left internal carotid artery (ICA) through the ECA, then advanced $3 \mathrm{~mm}$ past the bifurcation of the left ICA, until resistance was felt. Sutures were withdrawn and ICA patency confirmed. In the sham group, sutures were not advanced past the internal carotid artery bifurcation, identical to other procedures.

\section{Intracerebroventricular Injection}

The operation of intracerebroventricular injection was as previously described (13). A hole, lateral to the bregma $1.0 \mathrm{~mm}$, was drilled on the skull without puncturing the dura. A needle of a 10- $\mu$ L Hamilton syringe (Microliter 701; Hamilton Company, Reno, NV, USA) was then stereotactically and vertically inserted into the hole, $3.0 \mathrm{~mm}$ under the horizontal plane of the bregma, entering the left lateral ventricle. A total $2 \mu \mathrm{L}$ volume of recombinant NEK7 protein solution (Sino Biological, Beijing, China, $4 \mu \mathrm{g}$ dissolved in $2 \mu \mathrm{L}$ sterile saline), nigericin solution (Sigma-Aldrich, Shanghai, China, $4 \mu \mathrm{g}$ in $2 \mu \mathrm{L}$ vehicle, vehicle: $2 \mu \mathrm{L}$ mixture of 9:1 NS and DMSO), or MCC950 solution (Sigma-Aldrich, Shanghai, China, $2 \mu \mathrm{g}$ in $2 \mu \mathrm{L}$ vehicle, vehicle: $2 \mu \mathrm{L}$ mixture of 9:1 NS and DMSO) was infused at a rate of
$0.2 \mu \mathrm{L} / \mathrm{min}, 1 \mathrm{~h}$ after $\mathrm{SAH}$ induction, whereas $500 \mathrm{pmol} / 2 \mu \mathrm{L}$ of NEK7 siRNA (Santa Cruz Biotechnology, Santa Cruz, CA, USA, $500 \mathrm{pmol}$ in a $2-\mu \mathrm{L}$ mixture of 1:1 RNase-free water and lipofectamine) or scrambled siRNA was infused at the identical rate $48 \mathrm{~h}$ before $\mathrm{SAH}$ induction. The syringe was slowly withdrawn at $10 \mathrm{~min}$ after infusion.

\section{Neurological Outcome Assessment}

Neurological deficits were accessed as previously described, at 24 and $72 \mathrm{~h}$ after SAH induction. The modified Garcia scale and beam balance test were used (14). Two blinded observers were employed for grading mean of the neurological score.

\section{SAH Grade}

The SAH severity grading score was implemented as previously described (15). The basal cistern was divided into six segments that could be scored from 0 to 3 according to the amount of subarachnoid blood clotting. The total score was calculated by adding the scores from six segments ( $0-18$ points). Animals that received a score $<8$ were excluded from the study.

\section{Brain Water Content}

The brain samples were quickly removed from the skull, separated into the left and right cerebral hemispheres, the cerebellum, and the brain stem. These four parts of the brain were weighed (wet weight), respectively, and the brain samples were then dried at $55^{\circ} \mathrm{C}$ for $48 \mathrm{~h}$ in an oven and weighed again (dry weight). The water content percentage formula was: ([wet weight - dry weight]/wet weight) $\times 100 \%$.

\section{Evans Blue Extravasation}

Evans blue extravasation was performed as previously described (14). Mice were anesthetized by pentobarbital sodium $(40 \mathrm{mg} / \mathrm{kg})$ i.p. injection $24 \mathrm{~h}$ post-SAH. Evans blue dye $(2 \%, 5 \mathrm{~mL} / \mathrm{kg}$; Sigma-Aldrich, St. Louis, MO, USA) was injected into the left femoral vein over $2 \mathrm{~min}$ and circulated for $60 \mathrm{~min}$. Mice were euthanized by an intracardial perfusion of phosphate-buffered saline (PBS). The brains were removed and quickly divided into the left and right cerebral hemispheres, weighed, homogenized in saline, and centrifuged at $15,000 \mathrm{~g}$ for $30 \mathrm{~min}$. Subsequently, the resultant supernatant was added with equal volume of trichloroacetic acid, incubated overnight at $4^{\circ} \mathrm{C}$ and centrifuged at $15,000 \mathrm{~g}$ for $30 \mathrm{~min}$. Next, the resultant supernatant was collected and spectrophotometrically quantified at $610 \mathrm{~nm}$ for Evans blue dye.

\section{Immunofluorescence Staining}

Immunofluorescence staining was performed on fixed frozen brain sections as previously described $(16,17)$. Mice were deeply anesthetized and transcardially perfused with PBS and $4 \%$ PFA at $24 \mathrm{~h}$ post SAH. Brain samples were isolated and post-fixed in $4 \%$ PFA for $24 \mathrm{~h}$, then soaked in $40 \%$ sucrose for 1 day. Coronal brain sections $(10 \mu \mathrm{m})$ were obtained using a cryostat (Leica, Nussloch, Eisfeld, Germany) and permeabilized using $0.3 \%$ Triton X-100 in PBS for $30 \mathrm{~min}$. Sections were blocked with $5 \%$ donkey serum for 1 and incubated at $4{ }^{\circ} \mathrm{C}$ overnight with anti-Occludin (1: 100, Abcam, Cambridge, UK), anti-NEK7 (1: 200, Novus, Bio-Techne China, Shanghai, 
China), anti-IL-1 $\beta$, anti-NeuN, anti-GFAP (1: 100), anti-Iba1, anti-NG2 (1: 50, Abcam, Cambridge, UK) antibody and Lectin (1:200, Vector Laboratories, Burlingame, CA, USA), then incubated with corresponding secondary antibodies for $4 \mathrm{~h}$ at room temperature. Images were obtained atbasal cortex with a fluorescence microscope (Olympus, Melville, NY, USA) from slides. Quantification of immunofluorescence intensity was performed with the Measure tool in ImageJ software (National Institutes of Health, Bethesda, MD, USA) and the results of all the groups were normalized to the Sham group. Six image fields per group were analyzed. To evaluate the continuity of tight junctions of ECs, gaps of tight junctions were quantified as the length percentage of whole Occludin staining as per the previous method (18). The length of gaps and whole Occludin staining was measured by the Line tool in ImageJ software (National Institutes of Health, Bethesda, MD, USA) and the percentage was calculated as follows: Gap formation percentage = gap length/whole Occludin length. At least six vessels in each group were analyzed.

\section{TUNEL}

TUNEL staining was performed to test neuron apoptosis. Paraffin-embedded left/ ipsilateral hemisphere basal cortex sections $(10 \mathrm{~mm})$ were prepared. Apoptotic cells were stained using a Roche in situ cell death detection kit (Roche, Basel, Switzerland) according to the manufacturer's instructions. Fluorescent pictures were captured using a fluorescence microscope (Olympus, Melville, NY, USA). The number of TUNEL-positive cells were counted with ImageJ software. To calculate the apoptotic rates, six slides from one group were chosen and the number of TUNEL+ cells were counted in the range of images. The apoptotic rates were calculated by the formula: apoptotic rates $=$ the number of TUNEL + neurons in the field/ the number of all neurons in the field $\times 100 \%$.

\section{Western Blot}

Western blot was performed as previously described $(19,20)$. Briefly, a protein sample was extracted from the cortex of the left/ipsilateral hemisphere. Equal amounts of total protein $(30 \mu \mathrm{g})$ were loaded in the lane of SDS-PAGE gels. After electrophoresis, the protein was transferred onto a PVDF membrane, which was then blocked with blocking buffer for $2 \mathrm{~h}$ at room temperature. Subsequently, the membrane was incubated at $4{ }^{\circ} \mathrm{C}$ overnight with the following antibodies: antiNEK7 (1:500; Novus, Bio-Techne China, Shanghai, China), antiNLRP3, anti-ASC (1:500; Abcam, Cambridge, UK), anti-caspase1, anti-IL-1 $\beta$ (1:500; Santa Cruz Biotechnology, Santa Cruz, CA, USA), anti-BCL-2, and anti-BAX (1:500; Thermo Fisher Scientific, Waltham, MA, USA). $\beta$-actin and $\alpha$-tubulin were used as internal loading controls, anti- $\beta$-actin, and anti- $\alpha$-tubulin primary antibodies (1:2000; Santa Cruz Biotechnology, Santa Cruz, CA, USA). PVDF membranes were incubated with the appropriate secondary antibodies for $2 \mathrm{~h}$ at room temperature. Blots were detected by chemiluminescent (ECL Plus; Amersham Bioscience, Arlington Heights, IL, USA). Data were analyzed by densitometry using Quantity One 4.6.2 (Bio-Rad Laboratories, Berkeley, CA, USA).

\section{Statistical Analysis}

Data are shown as the mean \pm SD. Chi-square tests and one-way ANOVA, followed by Tukey's multiple comparisons test were used to compare the different groups. SPSS 18 software (IBM, Chicago, IL, USA) was utilized to analyze the data, and $P<0.05$ was considered as statistically significant.

\section{RESULTS}

\section{Expression of NEK7 and Cellular Distribution After SAH}

To investigate the expression of NEK7 after SAH induction, protein levels of NEK7 was detected by Western blot analysis. A significant increase of NEK7 were found in the ipsilateral/left hemisphere at 24 and $72 \mathrm{~h}$ after SAH (Figures 1A,B). Furthermore, immunohistochemical staining in the sham group showed that NEK7 expression was colocalized with the NeuN (neuron marker), GFAP (astrocyte marker), Lectin (endotheliocyte marker), Iba-1 (microglia marker), and nerve/glial antigen (NG) 2 (oligodendrocyte marker) in the left/ipsilateral cortex (Figure 1C). At $24 \mathrm{~h}$ post SAH, NEK7 was obviously elevated in endothelial cells and microglia (Figures 1D,E). None of the sham-operated mice died, and eight mice died within $72 \mathrm{~h}$ after $\mathrm{SAH}$, due to severe hemorrhagic volume.

\section{Neurological Functions After Recombinant NEK7 Delivery and Interference of NEK7 Expression After SAH}

The NEK7 siRNA used in the present study could effectively inhibit the NEK7 expression after SAH. SAH grading score did not display significant differences among the groups at 24 and $72 \mathrm{~h}$ after SAH (Figures 2A,B). The SAH group showed significant neurological impairment in the modified Garcia test and beam balance, compared with the Sham group at $24 \mathrm{~h}$ after SAH induction (Figures 2C-F). Mice in the SAH+NEK7 $100 \mathrm{ng} / \mu \mathrm{L}$ group had more severe neurological deficits at $24 \mathrm{~h}$ post $\mathrm{SAH}$ compared with the $\mathrm{SAH}+\mathrm{NS}$ group, different to that in the SAH+NEK7 $5 \mathrm{ng} / \mu \mathrm{L}$ and SAH+NEK7 $25 \mathrm{ng}$ group (Figures 2C-F). Furthermore, NEK7 siRNA treatment revealed greater improvements in neurological deficits compared with the $\mathrm{SAH}+\mathrm{Scr}$ siRNA group at $24 \mathrm{~h}$ after SAH (Figures $\mathbf{2 C - F}$ ). The Beam balance test exhibited similar trends (Figures 2C-F).

\section{Neuronal Apoptosis and BBB Permeability After Recombinant NEK7 Delivery and Interference of NEK7 Expression}

Mice from the SAH, SAH+NS, and SAH+Scr siRNA groups showed increased brain water content in both hemispheres at 24 and $72 \mathrm{~h}$ after $\mathrm{SAH}$ compared with mice in the Sham group (Figures 3A,B). The NEK7 $100 \mathrm{ng} / \mu \mathrm{L}$ treatment increased the brain water content compared with the SAH+NS group, while the NEK7 siRNA pretreatment significantly reduced the 


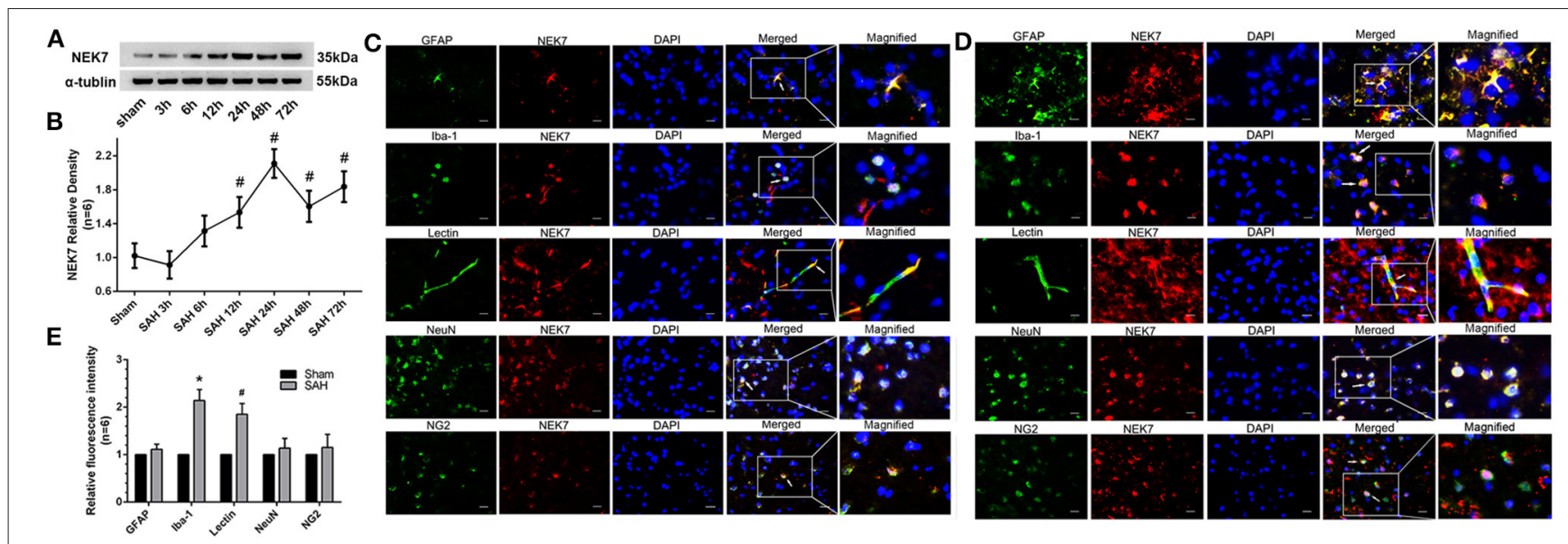

FIGURE 1 | Time course of endogenous NEK7 and cell type location after subarachnoid hemorrhage. (A,B) Representative western blot bands and quantitative analysis of NEK7 from the ipsilateral cortex after $\mathrm{SAH}, n=6$. Relative densities of each protein have been normalized against the sham group. \#: vs. sham $P<0.05$. (C,D) Representative immunohistochemistry slices of NEK7 (red), GFAP, Iba-1, Lectin, NeuN, NG2, (green) in the sham and SAH group at $24 \mathrm{~h}$ after SAH, $n=1$. Scale Bar $=10 \mu \mathrm{m}$. (E) Quantitative analysis of NEK7 fluorescent intensity in each type of cell. *: vs. sham (lba-1) $P<0.05$, \#: vs. sham (Lectin) $P<0.05$.

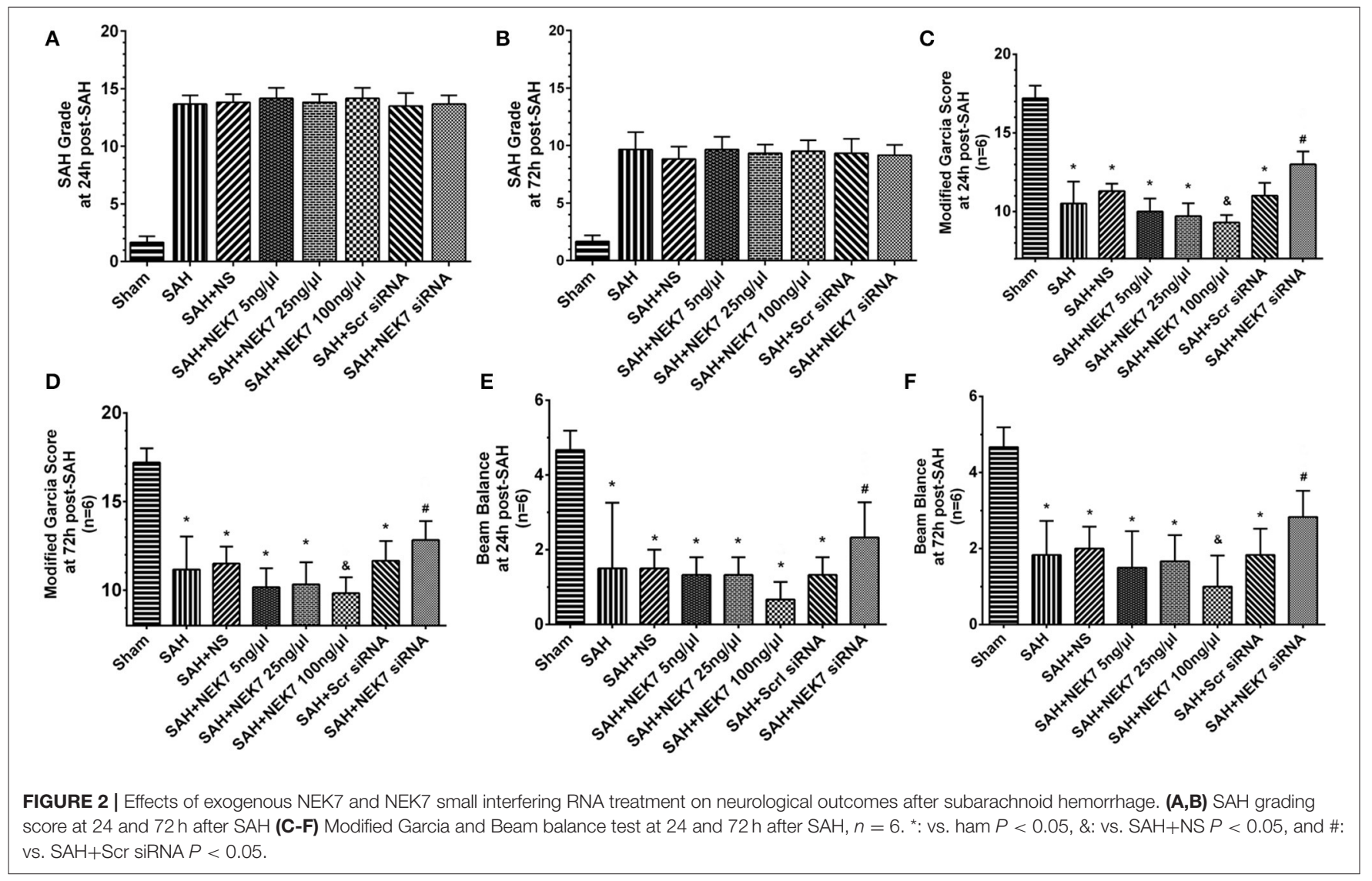

brain water content in both hemispheres at 24 and $72 \mathrm{~h}$ post SAH (Figures 3A,B). Therefore, a dosage of NEK7 100 $\mathrm{ng} / \mu \mathrm{L}$ was chosen for further investigation. Apoptosis is a biologically reversible process which is characterized as energydependent programmed cell death (21). Neuronal cell apoptosis, a main process of EBI, plays a crucial role in pathophysiology after SAH (22). Mice in the SAH and SAH+NS group showed a significant increase of neuronal apoptosis in the left/ipsilateral cortex. In the SAH+NEK7 $100 \mathrm{ng} / \mu \mathrm{L}$ group, mice exhibited a rising number of apoptosis neurons than that in the SAH+NS group, while NEK7 siRNA pretreatment reversed these phenomena (Figures 3C,D). Furthermore, the 


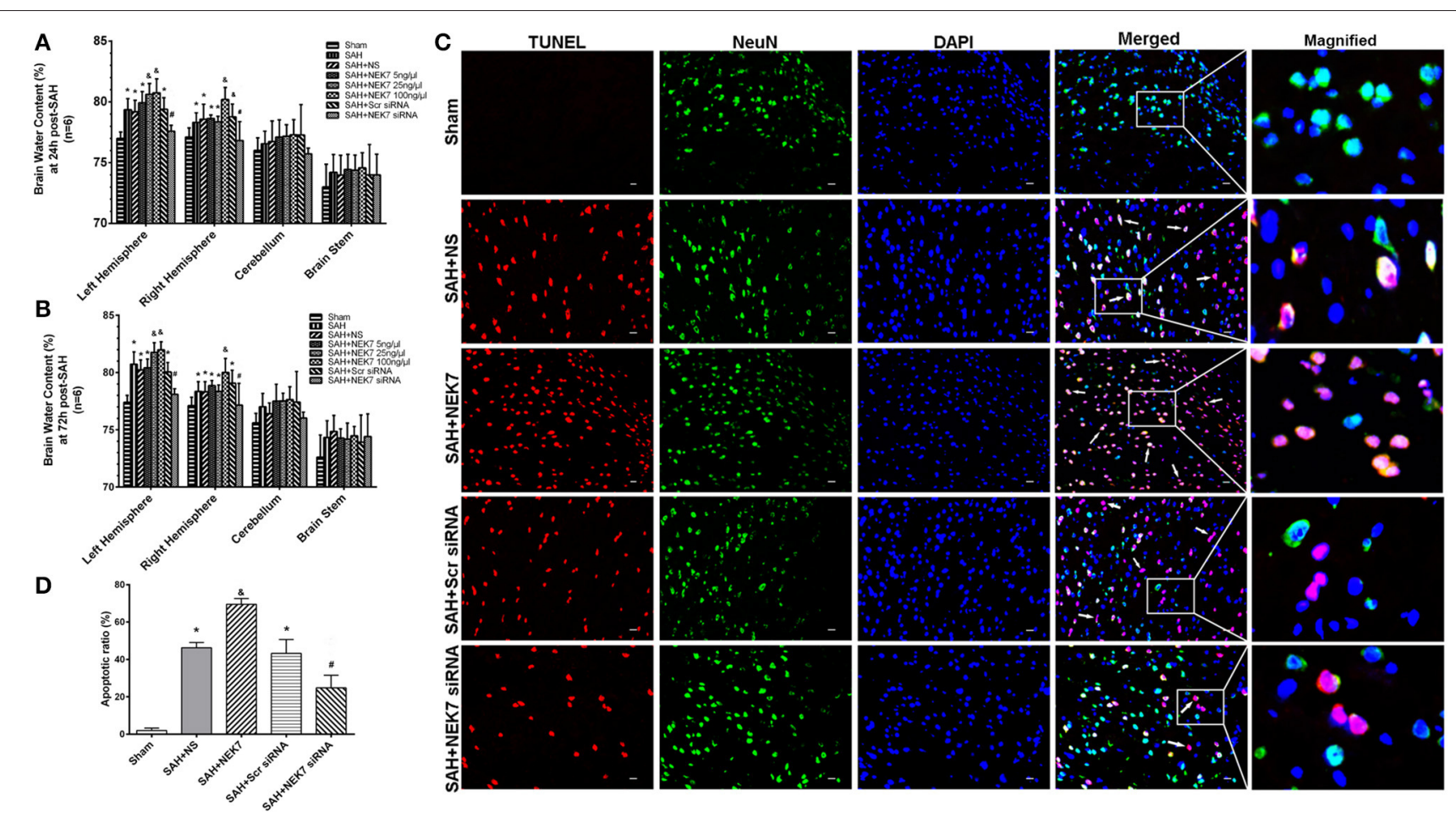

FIGURE 3 | Effects of exogenous NEK7 and NEK7 small interfering RNA treatment on neuronal apoptosis after subarachnoid hemorrhage. (A,B) Brain water content assessment at 24 and $72 \mathrm{~h}$ after SAH, $n=6$. (C) Representative immunohistochemistry images of TUNEL (red) and NeuN (green) at $24 \mathrm{~h}$ after SAH. Scale Bar $=$ $20 \mu \mathrm{m}$. (D) Apoptotic ratios of each group, $n=6$. *: vs. sham $P<0.05$, \&: vs. SAH+NS $P<0.05$, and \#: vs. SAH+Scr siRNA $P<0.05$.

SAH group demonstrated more Evans blue extravasation than the Sham group in the left/ipsilateral hemisphere (Figure 4A). The SAH+NEK7 $100 \mathrm{ng} / \mu \mathrm{L}$ mice exhibited increased Evans blue leakage compared with the SAH+NS mice, whereas the NEK7 siRNA pretreatment significantly lowered the Evans blue extravasation compared with the $\mathrm{SAH}+\mathrm{Scr}$ siRNA group (Figure 4A). In the immunohistochemical staining images, continuous endothelial cells (Lectin) and Occludin in the sham group were shown. Th Lectin and Occludin continuous structures broke up at $24 \mathrm{~h}$ after SAH, and NEK7 treatment significantly aggravated those damages, however, NEK7 siRNA infusion significantly reversed those disruptions at $24 \mathrm{~h}$ after $\mathrm{SAH}$ (Figures $4 \mathrm{~B}, \mathrm{C}$ ). There was a significant accumulation of microglia in the SAH+NS group compared to that in the sham group (Figure 4D). IL-1 $\beta$ expression in microglia was elevated in the SAH+NS group after SAH and was aggravated by NEK7 infusion, while NEK7 siRNA administration reversed those effects (Figure 4E).

\section{NLRP3 Inflammasome Protein and Apoptosis Related Protein Alteration After Recombinant NEK7 Delivery and Specific Inhibition of NEK7 Expression}

Expression of NEK7, NLRP3, ASC, caspase-1, and IL-1 $\beta$ was measured via western blot at $24 \mathrm{~h}$ after SAH. NLRP3 levels in $\mathrm{SAH}, \mathrm{SAH}+\mathrm{NS}, \mathrm{SAH}+\mathrm{NEK} 7100 \mathrm{ng} / \mu \mathrm{L}, \mathrm{SAH}+\mathrm{Scr}$ siRNA, and
SAH+ NEK7 siRNA elevated significantly at $24 \mathrm{~h}$ post SAH (Figures 5A,B). NEK7 $100 \mathrm{ng} / \mu \mathrm{L}$ infusion after SAH increased ASC expression and caspase- 1 and IL- $1 \beta$ maturation, however, NEK7 siRNA pretreatment reduced mature caspase-1 and IL$1 \beta$ expression (Figures 5A-G). BCL-2 (B-cell lymphoma 2 ), is a member of the BCL-2 family of regulator proteins that inhibit apoptosis. It plays an important role in promoting cellular survival and inhibiting the actions of pro-apoptotic proteins (21). $\mathrm{BAX}$ (Bcl-2-associated $\mathrm{X}$ protein) is a pro-apoptotic protein in the BCL-2 family, which acts on the mitochondrial membrane to promote permeabilization, which is a vital signal in the apoptosis cascade (23). Mice from the SAH and SAH+NS groups showed significantly increased levels of BAX and decreased BCL2 (Figures 5H-J). In the SAH+NEK7 group, expression of BAX rose and BCL-2 reduced, compared to that in the SAH+NS group. While in the $\mathrm{SAH}+\mathrm{NEK} 7$ siRNA group, expression of BAX was lower, and BCL-2 was more than that in the SAH+Scr siRNA group (Figures $5 \mathbf{H}-\mathbf{J}$ ).

\section{Neurological Functions and Neuronal Apoptosis After Nigericin Delivery, Specific Inhibition of NEK7 Expression, and MCC950 Administration}

To study whether NLRP3 activation is dependent on NEK7 after SAH, we used nigericin, an activator of NEK7-dependent NLRP3 signal (10), or MCC950, an inhibitor of the NEK7/NLRP3 


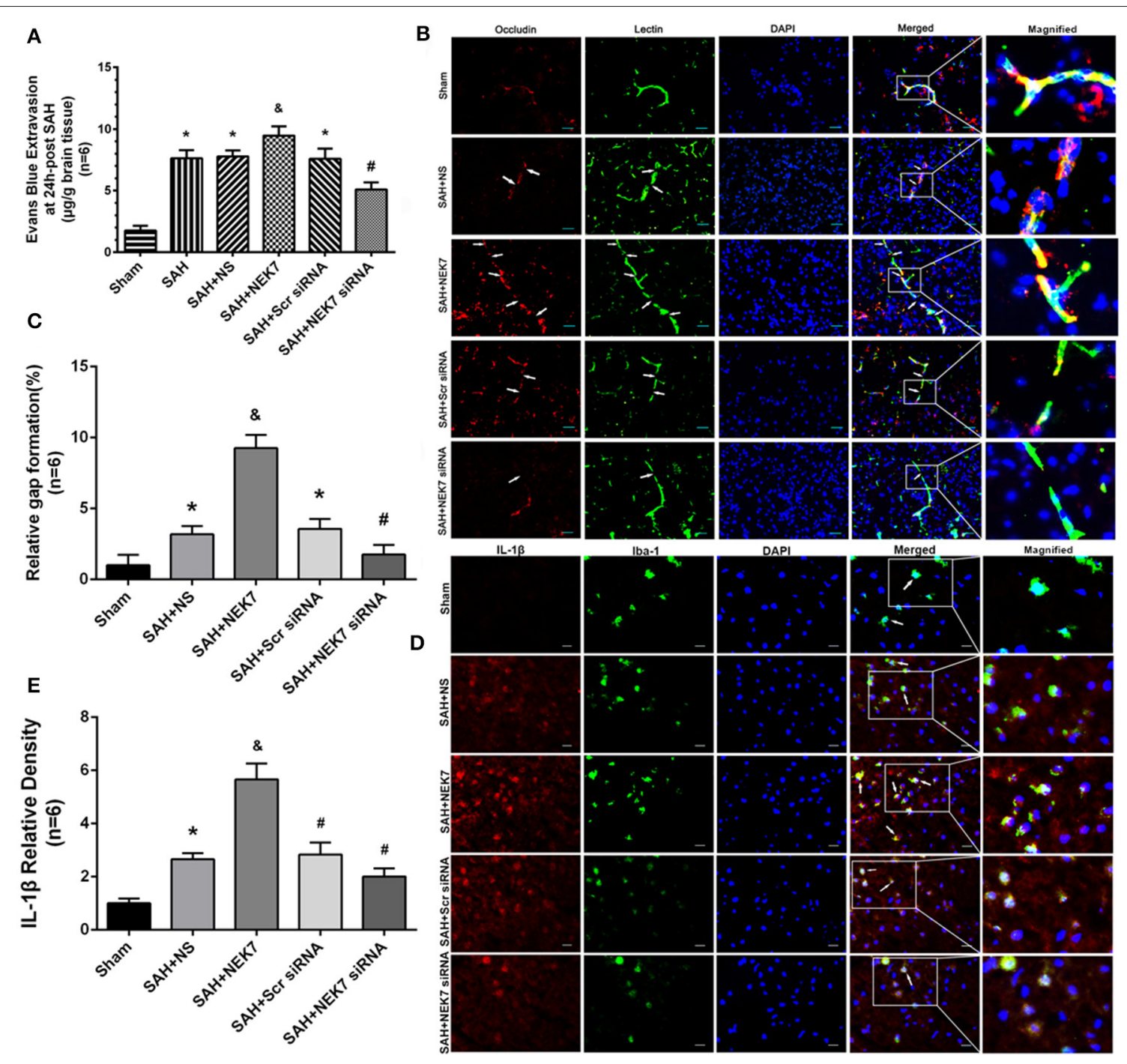

FIGURE 4 | Effects of exogenous NEK7 and NEK7 small interfering RNA treatment on blood-brain barrier and microglia accumulation after subarachnoid hemorrhage. (A) Evans blue extravasation evaluation at $24 \mathrm{~h}$ after SAH, $n=6$. (B) Representative immunohistochemistry images of Occludin (red) and Lectin (green) at $24 \mathrm{~h}$ after SAH. Arrow indicates the breakdown of continuous endothelia cell layer, $n=6$. Scale Bar $=20 \mu \mathrm{m}$. (C) Quantitative analysis of endothelial gap in (B). (D) Representative immunohistochemistry images of IL-1 $\beta$ (red) and Iba- 1 (green) at $24 \mathrm{~h}$ after SAH. Arrow indicates the accumulation of microglia and overlap of IL-1 $\beta$ and Iba- $1, n=6$. Scale Bar $=10 \mu \mathrm{m}$. (E) Quantitative analysis of IL-1 $\beta$ fluorescent in (D). *: vs. sham $P<0.05$, \&: vs. SAH+NS $P<0.05$, and \#: vs. SAH+Scr siRNA $P<0.05$.

signal (24), to regulate inflammation post SAH. The SAH grading score did not show significant differences among the groups at $24 \mathrm{~h}$ post $\mathrm{SAH}$ (Figure 6A). The $\mathrm{SAH}$ and $\mathrm{SAH}+$ Vehcile group showed significant neurological impairment in the modified Garcia score and beam balance test compared with the Sham group (Figures 6B,C). Mice in the SAH+negricin group had more severe neurological deficits at $24 \mathrm{~h}$ post $\mathrm{SAH}$ compared with the SAH+Vehicle group (Figures 6A-C). NEK7 siRNA treatment or MCC950 ameliorated neurological deficits, however, were comparable to that of the SAH+Scr siRNA group or SAH+Vehicle group, respectively (Figures 6A-C). Compared with the Sham group, the SAH+Vehicle group displayed obvious increasing apoptosis neuron numbers at the left/ipsilateral cortex at $24 \mathrm{~h}$ post SAH (Figures 6D,E). Nigericin administration increased neuronal apoptosis after SAH, while NEK7 knockdown or MCC950 injection reduced neuronal apoptosis (Figures 6D,E).

\section{BBB Permeability After Nigericin or MCC950 Delivery and Specific Inhibition of NEK7 Expression}

Mice in the SAH and SAH+Vehicle groups showed increased brain water content in left hemispheres $24 \mathrm{~h}$ after SAH, compared with the Sham group (Figure 7A). The nigericin treatment increased the brain water content compared with the SAH+ 


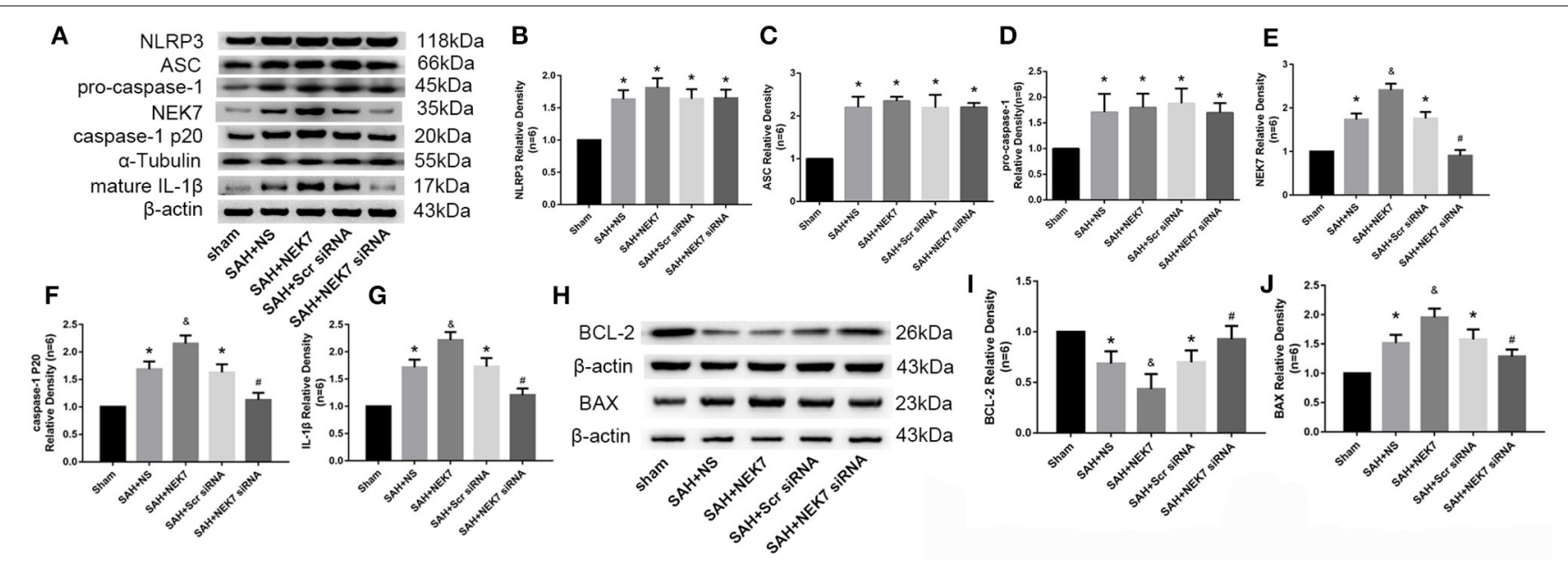

FIGURE 5 | Effects of exogenous NEK7 and NEK7 small interfering RNA pretreatment on NLRP3 activation and apoptosis related protein expression. (A) Representative western blot bands of NLRP3, NEK7, caspase-1, ASC, and IL-1 $\beta$ from the ipsilateral cortex after SAH. (B-G) Quantitative analysis of NLRP3, ASC, pro-caspase-1, caspase-1 p20, NEK7, and IL-1 $\beta, n=6$. (H) Representative western blot bands of BCL-2 and BAX. (I,J) Quantitative analysis of BCL-2 and BAX. Relative densities of each protein have been normalized to the sham group. $n=6$. *: vs. sham $P<0.05, \&$ : vs. SAH+NS $P<0.05$, and \#: vs. SAH+ scrambled SiRNA $P<0.05$

Vehicle group, however the NEK7 siRNA pretreatment or MCC950 injection significantly reduced the brain water content (Figure 7A). Furthermore, the SAH group showed more Evans blue extravasation than the Sham group (Figure 7B). The $\mathrm{SAH}+$ Nigericin group exhibited increased Evans blue leakage compared with the SAH+Vehicle group, whereas NEK7 siRNA pretreatment or MCC950 delivery significantly lowered the Evans blue extravasation compared with the SAH+Nigericin+Scr siRNA group (Figure 7B). Lectin and Occludin continuous structures broke up at $24 \mathrm{~h}$ after $\mathrm{SAH}$, and nigericin treatment significantly aggravated those damages, while NEK7 siRNA injection significantly reversed those disruptions (Figure 7C). Microglia accumulation was exacerbated and expression of IL-1 $\beta$ in microglia was elevated via nigericin administration and was alleviated after NEK7 siRNA pretreatment or MCC950 injection (Figures 7D,E).

\section{NEK7 Coordinated NLRP3 Signal Activation After SAH}

Expression of NEK7, NLRP3, ASC, pro-caspase-1, caspase-1 and IL- $1 \beta$ was measured via western blot at $24 \mathrm{~h}$ after SAH. NLRP3 was activated by various stimuli, and we wondered whether NEK7 coordinates NLRP3 activation after SAH. The expression of NEK7, NLRP3, caspase-1, and IL-1 $\beta$ was significantly increased at $24 \mathrm{~h}$ after SAH and NEK7 expression peaked at $24 \mathrm{~h}$ (Figures 5A-G). NEK7 recombinant protein delivery increased ASC oligomerization, caspase- 1 , and IL- $1 \beta$ maturation without increasing NLRP3 expression, and this was reversed by NEK7 siRNA infusion or MCC950 injection, which indicated that increasing NEK7 and NLRP3 binding activated the downstream signal (Figures 5A-G). BCL-2 protein levels in the $\mathrm{SAH}$ group were decreased at $24 \mathrm{~h}$ after $\mathrm{SAH}$ compared to the Sham group, while BAX increased and BCL2 decreased in the SAH+Vehicle group compared to the sham group (Figures $5 \mathbf{H}-\mathbf{J}$ ). NEK7 recombinant protein infusion lowered BCl-2 and elevated BAX, while NEK7 siRNA relieved those effects (Figures 5H-J). Nigericin injection increased the expression of caspase-1, IL- $1 \beta$ at $24 \mathrm{~h}$ after SAH, which demonstrated that nigericin increased caspase- 1 and IL- $1 \beta$ maturation but did not affect NLRP3 and NEK7 expression (Figures 8A-G,K-Q). Caspase-1 and IL-1 $\beta$ maturation were decreased at $24 \mathrm{~h}$ after $\mathrm{SAH}$ in the $\mathrm{SAH}+$ nigericin $+\mathrm{Scr}$ siRNA group compared to that in the $\mathrm{SAH}+$ nigericin+NEK7 siRNA group (Figures $\mathbf{8 A - G}, \mathbf{K}-\mathbf{Q}$ ). Nigericin treatment could effectively inhibit their BCL-2 expression and enhance BAX expression (Figures 8H-J), however NEK7 siRNA treatment (Figures 8H-J) or MCC950 administration significantly reversed those trends (Figures 8R-T).

\section{DISCUSSION}

The present study demonstrated that NEK7 increased after $\mathrm{SAH}$, peaked at $24 \mathrm{~h}$, and was mainly expressed in microglia and endothelial cells. Exogenous NEK7 protein worsened neurological deficits and brain edema, while NEK7 specific inhibition preserved neurological function, neuronal apoptosis, and brain edema. Administration of Nigericin, a NEK7dependent NLRP3 activator, aggravated neurological deficits, brain edema, microglial accumulation, and BBB disruption, while NEK7 siRNA infusion reversed these phenomena. By further studying the mechanism it was indicated that NEK7 activated the NLRP3 downstream inflammatory pathway, including caspase-1 and IL-1 $\beta$ maturation in endothelial cells which open, leading to increasing BBB extravasation. The microglia were then activated, which subsequently induced neuronal apoptosis by reducing BCL-2 and increasing BAX. NEK7 was recently reported to be critical for the activation of NLRP3 inflammasome and its 


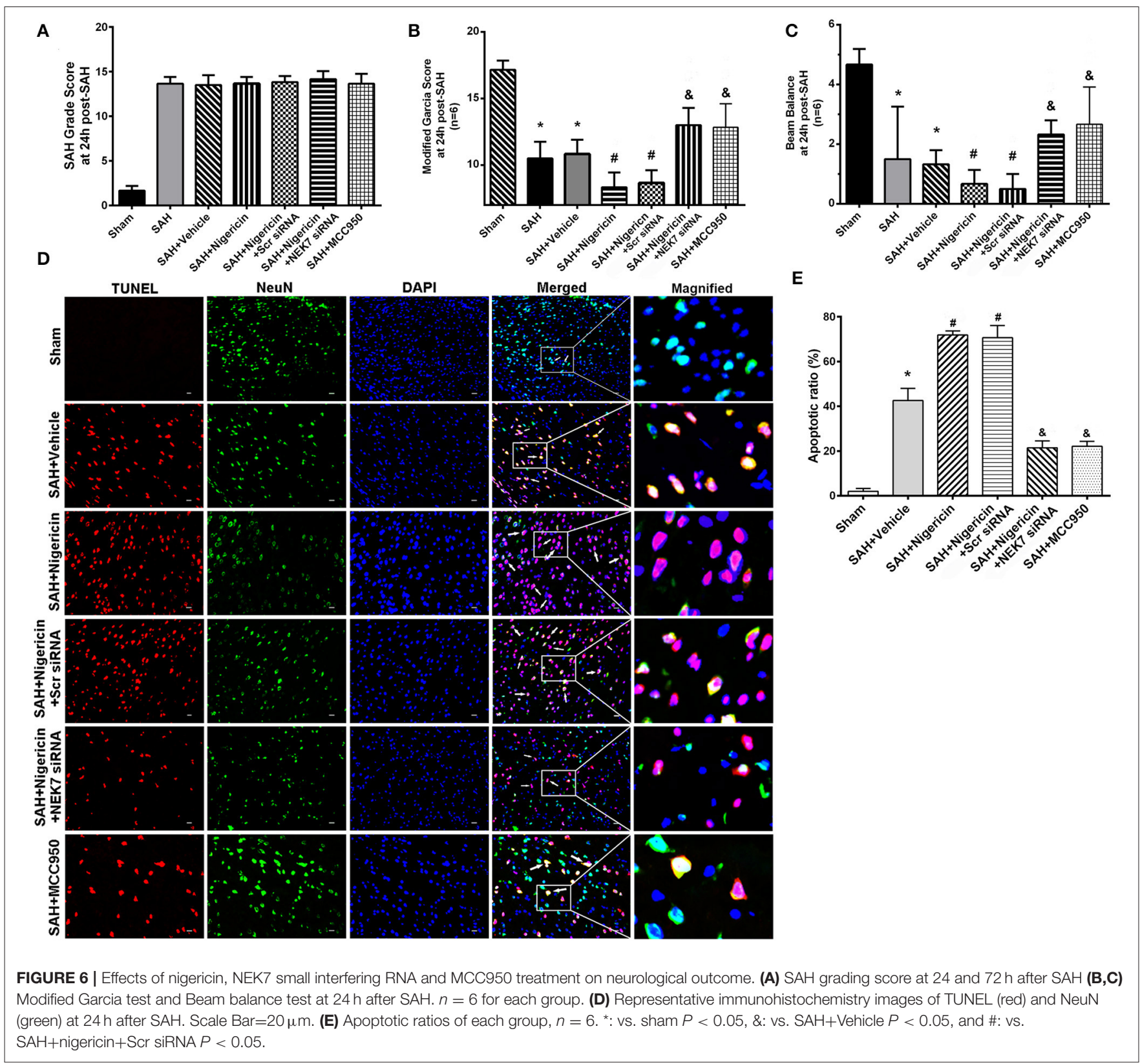

downstream inflammatory pathway, to induce caspase- 1 and IL- $1 \beta$ maturation $(4,10,25)$. NLRP3 inflammasome, which is important for the activation of caspases-1, maturation, and the secretion of proinflammatory cytokines such as IL-1 $\beta$ and IL-18, is a complex with multi-protein components containing three parts: an amino-terminal pyrin domain, a central nucleotidebinding domain, and a C-terminal leucine rich repeat (26). The ASC, and pro-caspase- 1 are closely linked with the formation of NLRP3 inflammasome (27). Under the pathological state, NLRP3 is activated, then inflammasome assembly takes place and leads to the IL- $1 \beta$ shearing from pro-IL- $1 \beta$, then secretion and maturation, which finally causes cell injury and death (7). NLRP3 inflammasome is involved in the initiation and development of several pathophysiological processes of CNS injury including stroke (28), neurodegenerative disease (7), and multiple sclerosis (29).

NEKs (NIMA-related kinase) are a group of evolutionarily conserved protein kinases sharing high amino acid sequence identity with NIMA which controls initiation of cell mitosis during mitotic cell division (30). A decrease in the centrosomal ctubulin levels and reduction of the microtubule re-growth activity in the NEK7-suppressed cells was observed (31). The NEK7 amino acid sequence contains all of the amino acids conserved in serine/threonine kinases and have no C-terminal extensions (32), is expressed widely in a mouse brain including the Olfactory bulb, cortex, Thalamus, Midbrain, and the Hindbrain 

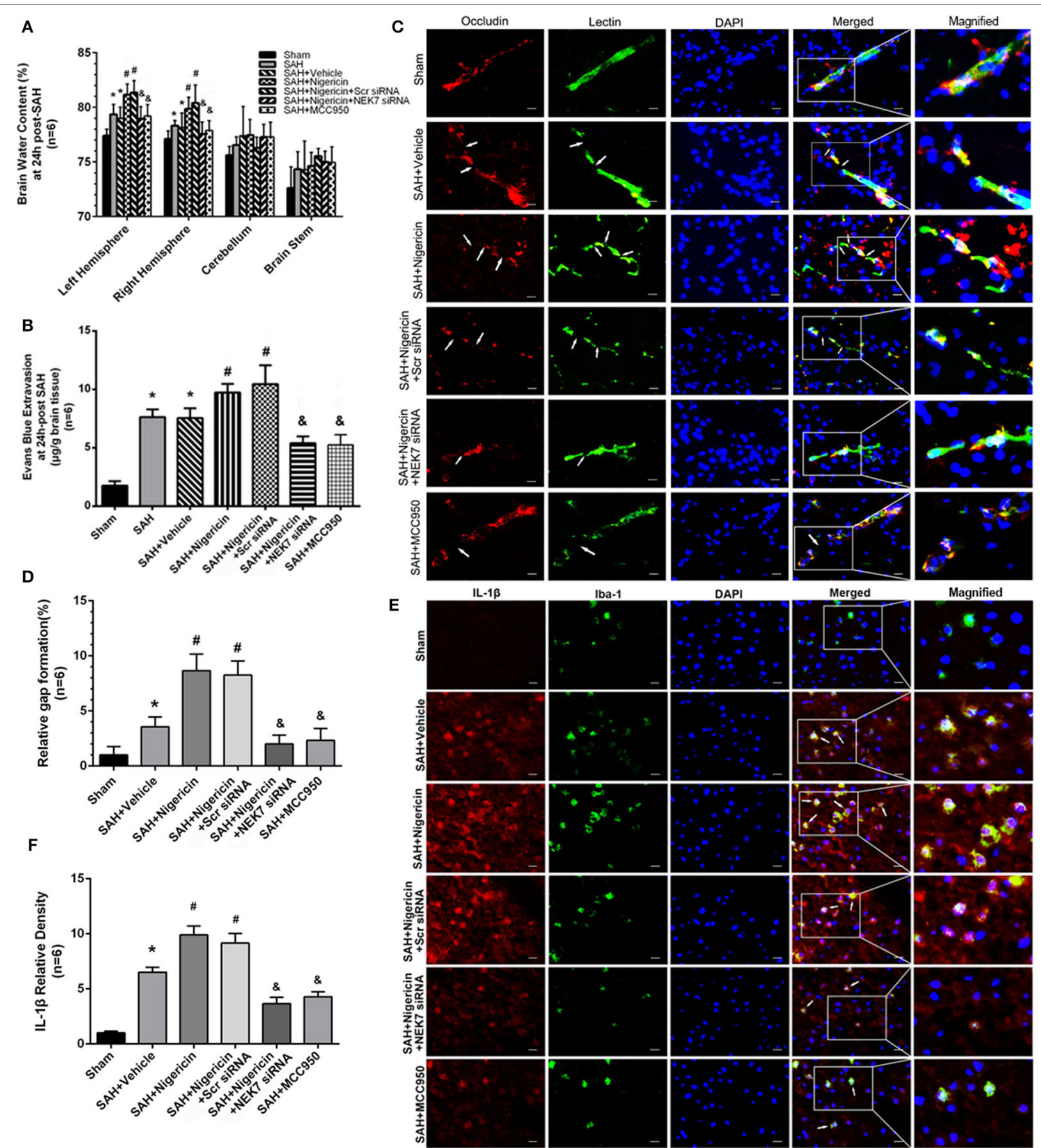

DAPI

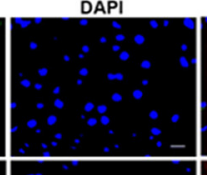

Merged

Magnified
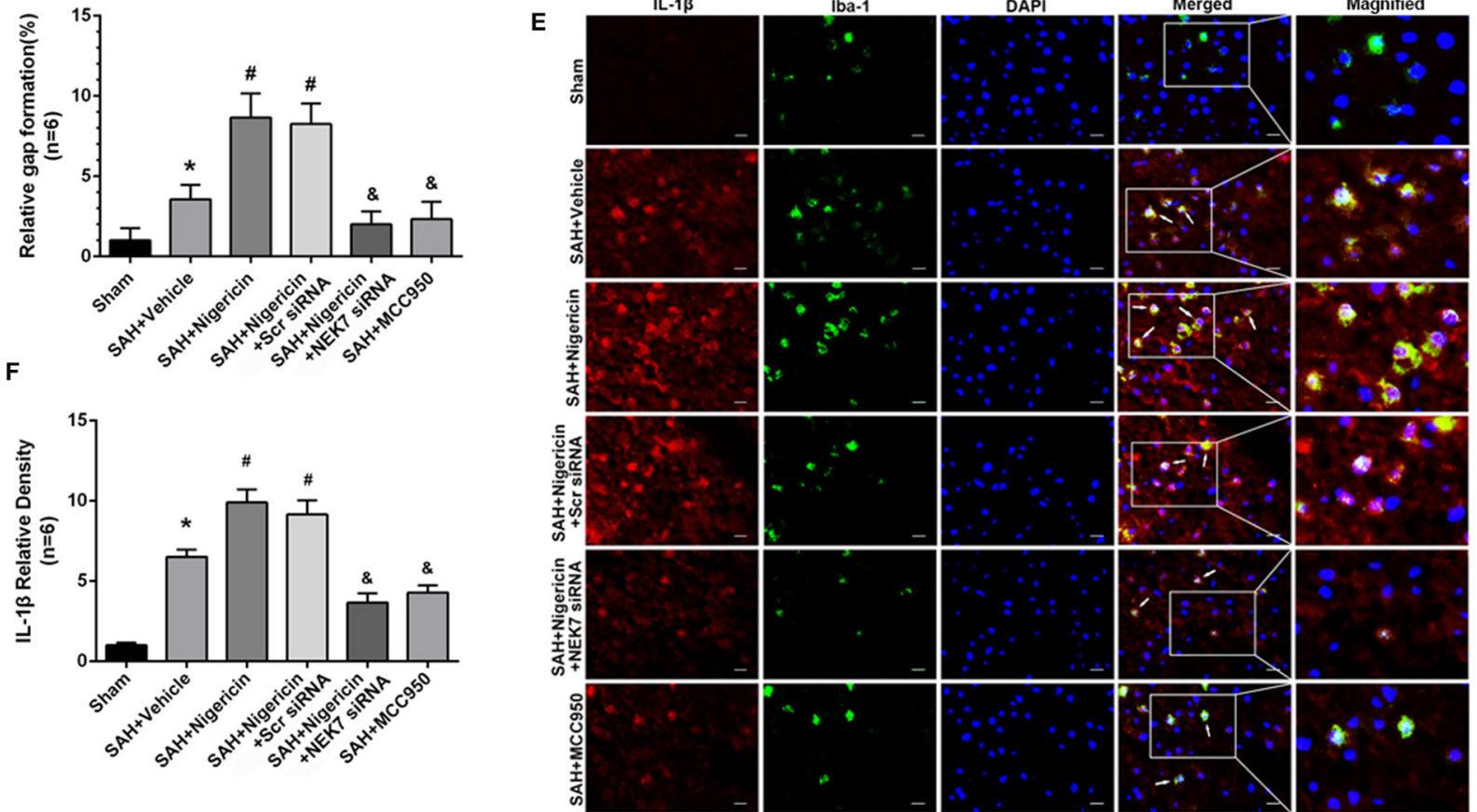

FIGURE 7 | Effects of nigericin, NEK7 small interfering RNA and MCC950 treatment on blood-brain barrier and microglia accumulation after subarachnoid hemorrhage. (A) Brain water content assessment at $24 \mathrm{~h}$ after $\mathrm{SAH}, n=6$. (B) Evans blue extravasation evaluation at $24 \mathrm{~h}$ after $\mathrm{SAH}, n=6$. (C) Representative immunohistochemistry images of Occludin (red) and Lectin (green) at $24 \mathrm{~h}$ after SAH. Arrow indicates the breakdown of continuous endothelia cell layer, $n=6$. (D) Quantitative analysis of endothelial gap in (C). (E) Representative immunohistochemistry images of IL-1 $\beta$ (red) and Iba- 1 (green) at $24 \mathrm{~h}$ after SAH. Arrow indicates the accumulation of microglia and overlap of IL-1 $\beta, n=6$. Scale Bar $=10 \mu \mathrm{m}$. (F) Quantitative analysis of IL-1 $\beta$ intensity in (E). ${ }^{*}$ : vs. sham $P<0.05$, \&: vs. SAH+Vehicle $P<0.05$, and \#: vs. SAH+nigericin+Scr siRNA $P<0.05$.

(33), is considered to be a new inflammasome component, and is also a switch between mitosis and NLRP3 activation (11). A recent study showed that NEK7-mutant mice had a defective IL-1 $\beta$ production response to nigericin (11). NEK7 bound to the leucine-rich repeat domain of NLRP3 in a kinase-independent manner (25) and acted as a coordinator 


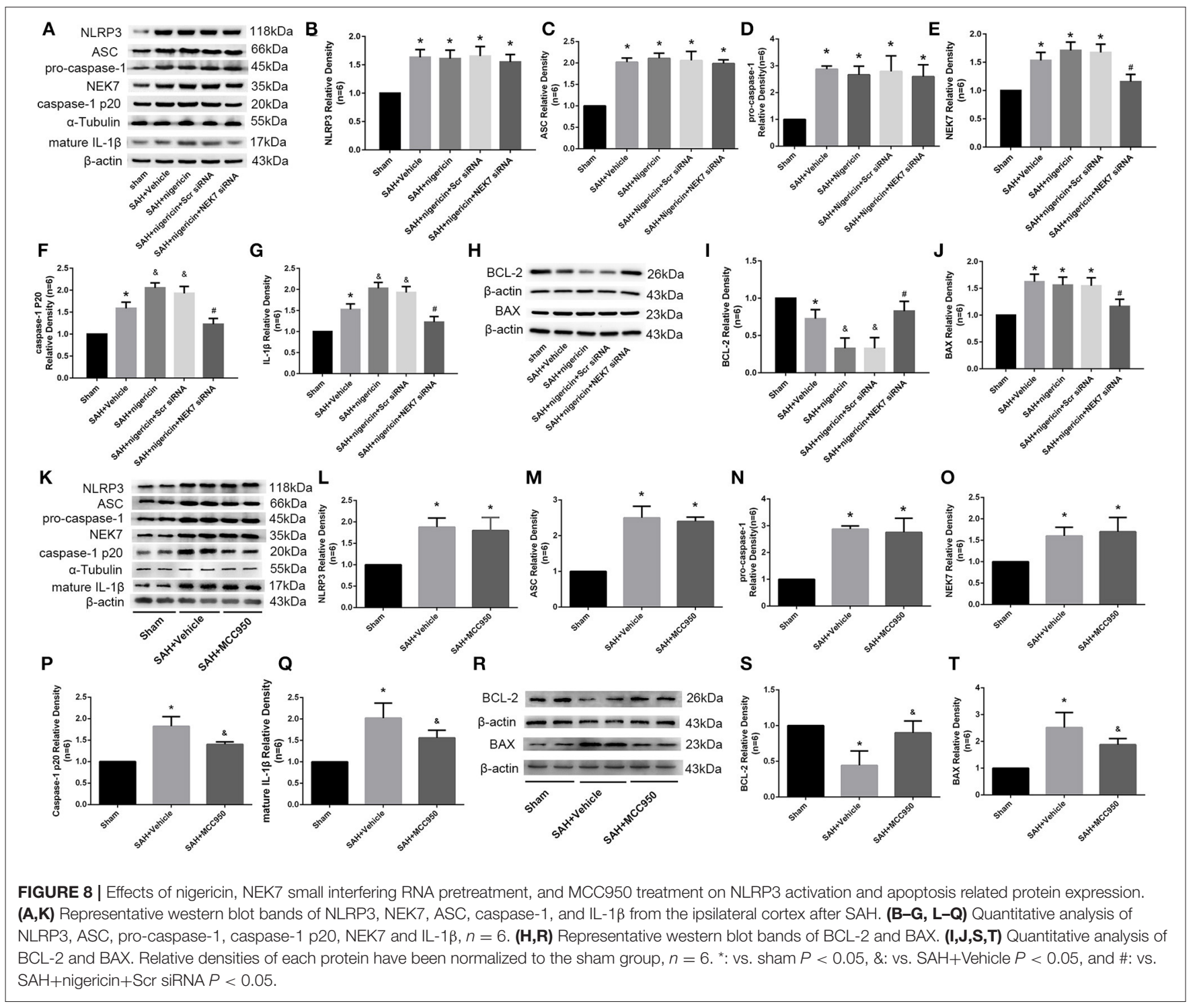

in NLRP3 inflammasome activation. NLRP3 inflammasome assembly and formation is required for NEK7, which results in the secretion and maturation of IL-1 $\beta$ and IL-18 (11). MCC950 is an orally bioavailable sulfonylurea derived small molecule which selectively interacts with NLRP3 with no effect on NLRC4 and NLRP1 (34), and a number of studies showed its ability to block NLRP3 activation through the NEK7/NLRP3 pathway under pathological conditions (24), however, the precise mechanism is unknown. Some studies have demonstrated that MCC950 could reduce lung ischemia-reperfusion injury and high level of glucose induced retina damage by blocking NEK7NLRP3 interaction $(35,36)$. Here we used MCC950 to block NEK7-NLRP3 pathway activation, and found that MCC950 showed similar effects as described in previous publications $(37,38)$. However, how MCC950 influences the NEK7-NLRP3 pathway still requires further study. Several pathways are pivotal for the NLRP3 inflammasome activation, such as potassium efflux, ROS (reactive oxygen species) signaling, and lysosomal destabilization. $50 \mathrm{mM} \mathrm{KCl}$ prevented potassium efflux to inhibit the interaction between NLRP3 and NEK7 induced by ATP, nigericin, or gramicidin in bone-marrow-derived macrophages from C57BL/6J mice carrying mutant NEK7 (25). While LPSprimed $\mathrm{NEK} 7^{+/+}$or $\mathrm{NEK} 7^{\mathrm{Cu} / \mathrm{Cu}}$ macrophages, producing similar amounts of mitochondrial ROS after treatment with nigericin (11). A recent pilot study found that hypokalemia is common in SAH patients (39). Cortical spreading depolarization, a phenomenon which is characterized as serial neuronal exciting via $\mathrm{K}^{+}$channel open after $\mathrm{SAH}$, was considered as a factor of poor prognosis (40). These studies suggested that $\mathrm{K}^{+}$efflux may exist after SAH and could be an activator of NEK7/NLRP3. Recently, NEK7 has been identified as a necessary component of the NLRP3 activation driven by ROS but is independent of K+ efflux (41). The upstream of NEK7/NLRP3 activation after SAH currently remains elusive and requires further investigation.

Microglia are a cluster of specialized macrophages residing in the CNS system and the main source of NLRP3 (42). 
Sufficient evidence suggests that they play a critical role in neuroinflammation. Activated microglia produce and secrete pro-inflammatory cytokines, including IL-1 $\beta$, nitric oxide, and tumor necrosis factor- $\alpha$, which induce cell death and secondary central nervous system injury. Cultured microglia have recently been shown to produce IL-1 $\beta$ (11), acting as a key mediator of neuronal apoptosis after SAH (43). In addition, Microglia were found to induce neuronal apoptosis via reducing BCL-2 and increasing BAX (44). In this study, we showed that NEK7 was expressed in microglia and was elevated after SAH, which in turn elicited neuronal apoptosis by activating NLRP3 and inducing IL-1 $\beta$ releasing. Neuronal apoptosis was alleviated by NEK7 siRNA pretreatment $v i a$ reducing IL- $1 \beta$ and BAX and increasing BCL-2. Microglial accumulation was also found after SAH, providing evidence that targeting NEK7 may reduce neurological deficits by attenuated neuronal apoptosis triggered by microglia activation. Our results displayed that microglial accumulation in the ipsilateral/left cortex was enhanced by exogenous NEK7 administration and nigericin infusion, while it was reversed by NEK7 siRNA pretreatment and MCC950 injection. NEK7 was reported to influence microtubule dynamics, which is also a main factor for cell movement, morphology maintaining, and synaptic outputs of neurons $(31,45,46)$. Microtubule activity were also found to be associated with microglia migration (47), however, there are very few studies that mention the exact mechanism of microglia movement after SAH. Based on these results, NEK7 may play a potential role in microglia migration via regulating microtubule. Furthermore, Acetylated $\alpha$-tubulin was recently found to mediate the transport of mitochondria, apposition of ASC to NLRP3 on endoplasmic reticulum, and then activated NLRP3 (48). This indicates that NEK7 might activate NLRP3 via activating microtubule acetylation after stroke, which requires further investigation.

The blood-brain barrier can prevent harmful substances from coming into contact with the brain and allows nutrient substances to enter the brain (49). After SAH, the bloodbrain barrier opens and leads to blood metabolites coming into direct contact with neurons and surrounding cells, which also leads to vasogenic brain edema (2). Endothelial cells are a main component of the BBB. Endothelial cells maintain the basic function of the $\mathrm{BBB}$ though transcellular transport and a tight junction structure. Previous studies indicated that NLRP3 activation induce the BBB open, while NLRP3 deletion preserved BBB permeability by reducing MMP9 and MMP2, which could subsequently degrade the tight junction protein including zonula occludens-1 and TJP2 (50). Expression of NLRP3 rise in endothelial cells after stroke, suggesting that endothelial cells may be a major source of $\operatorname{NLRP3}(36,50)$. Recent studies reveal that knockdown NEK7 expression could reduce high glucose induced retinal endothelial cell dysfunction (36) and maintain retinal endothelial cell permeability, which suggests that IL- $1 \beta$ itself could increase BBB permeability. In this investigation we demonstrated that brain edema worsened, and Evans blue extravasation increased after $\mathrm{SAH}$, and protein levels of IL-1 $\beta$ rose in endothelial cells. At the same time, knockdown NEK7 ameliorated downstream NLRP3 activation and IL-1 $\beta$ maturation, eventually protecting BBB.

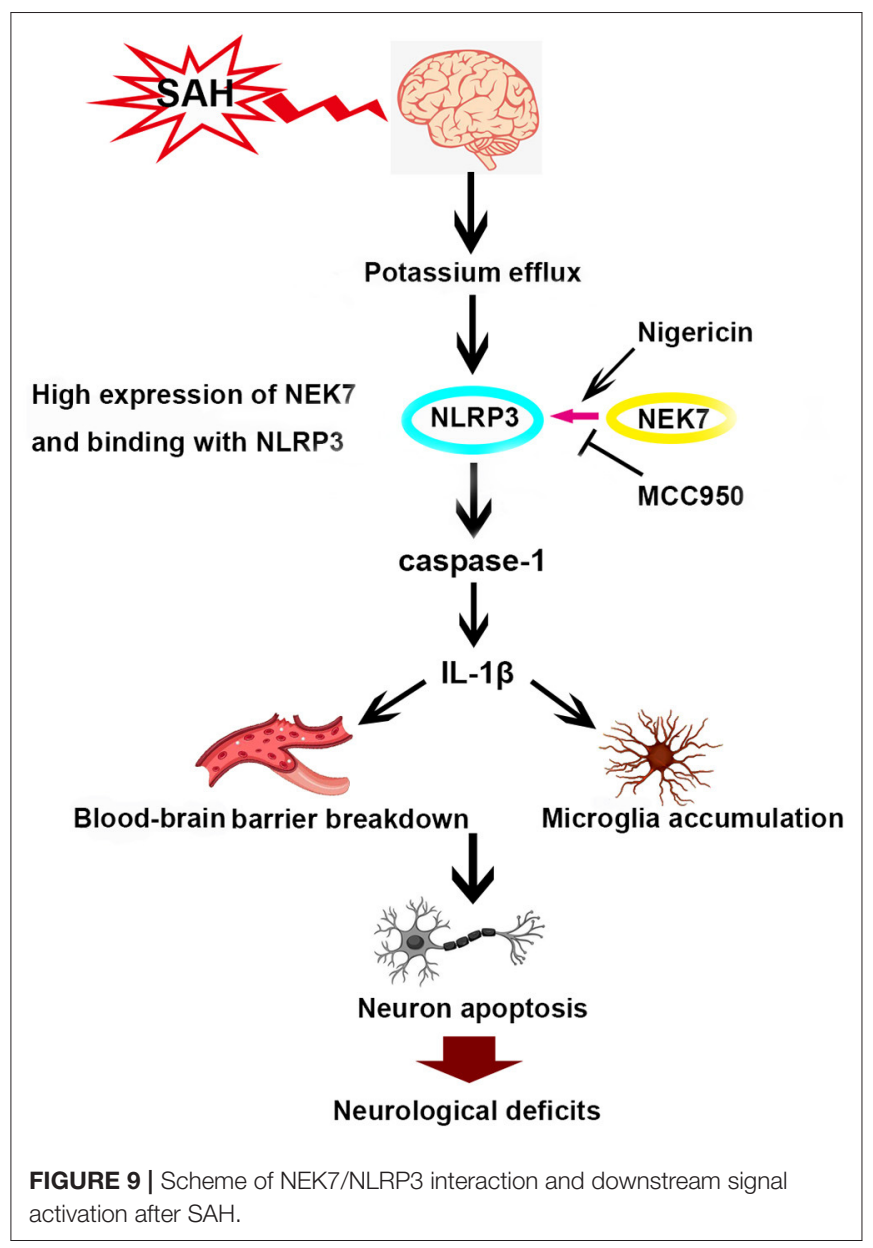

The limitations of this investigation were that data only focused on the NEK7 and NLRP3 inflammasome, while tight junction protein degradation was not studied in detail. After SAH, NF- $\kappa$ B undergoes nuclear translocation, and subsequently, elevated MMP9 transcription and induced basement membrane and tight junction protein degradation, which have all been well-illustrated in other studies. In addition, NEK7 may be located in the extracellular region and could affect surrounding cells. Consistent with previous studies, we found that endothelial cells and microglia highly expressed NEK7, however, whether NEK7-NLRP3 pathway activation in other cells and whether other types of cells secrete NEK7 and have effects on endothelial cells and microglia after SAH, are unknown. Other unknown mechanisms of NEK7 or NLRP3 activation cannot be excluded, therefore, in further studies, more data about the expression of NEK7 in other cell types and the elucidation of mechanisms of other mechanisms are needed.

\section{CONCLUSIONS}

This study demonstrated for the first time that NEK7 was elevated in the microglia and endothelial cells of the brain after SAH, and exogenous recombinant NEK7 induced 
neuronal apoptosis and blood-brain barrier disruption which subsequently worsens the neurological outcome. This process was potentially mediated by NEK7-NLRP3 activation, which may promote casepase- 1 and IL-1 $\beta$ maturation and secretion, and apoptosis related protein alteration in the brain (Figure 9). By targeting NEK7, our data and further translational studies may provide benefits for the management of SAH patients.

\section{DATA AVAILABILITY STATEMENT}

All datasets generated for this study are included in the article/supplementary material.

\section{ETHICS STATEMENT}

The animal study was reviewed and approved by Ethic Committee of General hospital of Northern Theater Command.

\section{REFERENCES}

1. Sehba FA, Hou J, Pluta RM, Zhang JH. The importance of early brain injury after subarachnoid hemorrhage. Progress Neurobiol. (2012) 97:1437. doi: 10.1016/j.pneurobio.2012.02.003

2. Zhang JH, Badaut J, Tang J, Obenaus A, Hartman R, Pearce WJ. The vascular neural network-a new paradigm in stroke pathophysiology. Nat Rev Neurol. (2012) 8:711-6. doi: 10.1038/nrneurol.2012.210

3. Shao BZ, Cao Q, Liu C. Targeting NLRP3 Inflammasome in the treatment of CNS diseases. Front Mol Neurosci. (2018) 11:320. doi: 10.3389/fnmol.2018.00320

4. Jourdan T, Godlewski G, Cinar R, Bertola A, Szanda G, Liu J, et al. Activation of the Nlrp3 inflammasome in infiltrating macrophages by endocannabinoids mediates beta cell loss in type 2 diabetes. Nat Med. (2013) 19:113240. doi: $10.1038 / \mathrm{nm} .3265$

5. Abdul-Muneer PM, Alikunju S, Mishra V, Schuetz H, Szlachetka AM, Burnham EL, et al. Activation of NLRP3 inflammasome by cholesterol crystals in alcohol consumption induces atherosclerotic lesions. Brain Behav Immun. (2017) 62:291-305. doi: 10.1016/j.bbi.2017.02.014

6. Yang G, Yeon SH, Lee HE, Kang HC, Cho YY, Lee HS, et al. Suppression of NLRP3 inflammasome by oral treatment with sulforaphane alleviates acute gouty inflammation. Rheumatology. (2018) 57:727-36. doi: 10.1093/rheumatology/kex499

7. Heneka MT, McManus RM, Latz E. Inflammasome signalling in brain function and neurodegenerative disease. Nat Rev Neurosci. (2018) 19:61021. doi: 10.1038/s41583-018-0055-7

8. Dong Y, Fan C, Hu W, Jiang S, Ma Z, Yan X, et al. Melatonin attenuated early brain injury induced by subarachnoid hemorrhage via regulating NLRP3 inflammasome and apoptosis signaling. J Pineal Res. (2016) 60:25362. doi: 10.1111/jpi.12300

9. Zhao H, Pan P, Yang Y, Ge H, Chen W, Qu J, et al. Endogenous hydrogen sulphide attenuates NLRP3 inflammasome-mediated neuroinflammation by suppressing the $\mathrm{P} 2 \mathrm{X} 7$ receptor after intracerebral haemorrhage in rats. $J$ Neuroinflammation. (2017) 14:163. doi: 10.1186/s12974-017-0940-4

10. Schmid-Burgk JL, Chauhan D, Schmidt T, Ebert TS, Reinhardt J, Endl E, et al. A Genome-wide CRISPR (Clustered Regularly Interspaced Short Palindromic Repeats) Screen Identifies NEK7 as an Essential Component of NLRP3 Inflammasome Activation. J Biol Chem. (2016) 291:1039. doi: 10.1074/jbc.C115.700492

11. Shi $H$, Wang $Y$, Li $X$, Zhan $X$, Tang $M$, Fina $M$, et al. NLRP3 activation and mitosis are mutually exclusive events coordinated by NEK7, a new inflammasome component. Nat Immunol. (2016) 17:2508. doi: 10.1038/ni.3333

\section{AUTHOR CONTRIBUTIONS}

YD, GLia, and PP designed the experiment. PP, GLi, DL, ZZ, and GH finished the experiments protocols. GLi and DL carried out data analysis. PP, YD, and GLia prepared and revised the manuscript and gave the final approval of manuscript to be published. All authors contributed to the article and approved the submitted version.

\section{FUNDING}

This work was supported by the National Natural Science Foundation of China (Grant Nos. 81671174, 81671313, 81971133), the LIAONING Science and Technology Project (20180550504, 20180550151), the Medical Science Youth Breeding Project of the Chinese People's Liberation Army (17QNP053), and the China Postdoctoral Science Foundation (2016M592951).
12. Li Q, Zhao $\mathrm{H}$, Pan $\mathrm{P}, \mathrm{Ru} \mathrm{X}$, Zuo $\mathrm{S}$, Qu J, et al. Nexilin regulates oligodendrocyte progenitor cell migration and remyelination and is negatively regulated by protease-activated receptor 1/Ras-Proximate1 signaling following subarachnoid hemorrhage. Front Neurol. (2018) 9:282. doi: 10.3389/fneur.2018.00282

13. Chen Y, Zhang Y, Tang J, Liu F, Hu Q, Luo C, et al. Norrin protected blood-brain barrier via frizzled-4/beta-catenin pathway after subarachnoid hemorrhage in rats. Stroke. (2015) 46:52936. doi: 10.1161/STROKEAHA.114.007265

14. Pan P, Zhang X, Li Q, Zhao H, Qu J, Zhang JH, et al. Cyclosporine A alleviated matrix metalloproteinase 9 associated blood-brain barrier disruption after subarachnoid hemorrhage in mice. Neurosci Lett. (2017) 649:7-13. doi: 10.1016/j.neulet.2017.03.050

15. Sugawara T, Ayer R, Jadhav V, Zhang JH. A new grading system evaluating bleeding scale in filament perforation subarachnoid hemorrhage rat model. $J$ Neurosci Methods. (2008) 167:327-34. doi: 10.1016/j.jneumeth.2007.08.004

16. Zuo S, Ge H, Li Q, Zhang X, Hu R, Hu S, et al. Artesunate protected bloodbrain barrier via sphingosine 1 phosphate receptor 1/phosphatidylinositol 3 kinase pathway after subarachnoid hemorrhage in rats. Mol Neurobiol. (2017) 54:1213-28. doi: 10.1007/s12035-016-9732-6

17. Li Q, Chen Y, Li B, Luo C, Zuo S, Liu X, et al. Hemoglobin induced NO/cGMP suppression deteriorate microcirculation via pericyte phenotype transformation after subarachnoid hemorrhage in rats. Sci Rep. (2016) 6:22070. doi: 10.1038/srep22070

18. Bauer AT, Burgers HF, Rabie T, Marti HH. Matrix metalloproteinase9 mediates hypoxia-induced vascular leakage in the brain via tight junction rearrangement. J Cereb Blood Flow Metab. (2010) 30:83748. doi: $10.1038 / j \mathrm{jbfm} .2009 .248$

19. Li B, He Y, Xu L, Hu Q, Tang J, Chen Y, et al. Progranulin reduced neuronal cell death by activation of sortilin 1 signaling pathways after subarachnoid hemorrhage in rats. Crit Care Med. (2015) 43:e30411. doi: 10.1097/CCM.0000000000001096

20. Li B, Luo C, Tang W, Chen Z, Li Q, Hu B, et al. Role of HCN channels in neuronal hyperexcitability after subarachnoid hemorrhage in rats. J Neurosci. (2012) 32:3164-75. doi: 10.1523/JNEUROSCI.5143-11.2012

21. Taylor RC, Cullen SP, Martin SJ. Apoptosis: controlled demolition at the cellular level. Nat Rev Mol Cell Biol. (2008) 9:231-41. doi: 10.1038/nrm2312

22. Chen S, Feng H, Sherchan P, Klebe D, Zhao G, Sun X, et al. Controversies and evolving new mechanisms in subarachnoid hemorrhage. Prog Neurobiol. (2014) 115:64-91. doi: 10.1016/j.pneurobio.2013. 09.002

23. Czabotar PE, Westphal D, Dewson G, Ma S, Hockings C, Fairlie WD, et al. Bax crystal structures reveal how $\mathrm{BH} 3$ domains activate $\mathrm{Bax}$ and 
nucleate its oligomerization to induce apoptosis. Cell. (2013) 152:51931. doi: 10.1016/j.cell.2012.12.031

24. Coll RC, Robertson AA, Chae JJ, Higgins SC, Munoz-Planillo R, Inserra $\mathrm{MC}$, et al. A small-molecule inhibitor of the NLRP3 inflammasome for the treatment of inflammatory diseases. Nat Med. (2015) 21:24855. doi: 10.1038/nm.3806

25. He Y, Zeng MY, Yang D, Motro B, Nunez G. NEK7 is an essential mediator of NLRP3 activation downstream of potassium efflux. Nature. (2016) 530:3547. doi: $10.1038 /$ nature 16959

26. Jha S, Ting JP. Inflammasome-associated nucleotide-binding domain, leucinerich repeat proteins and inflammatory diseases. J Immunol. (2009) 183:76239. doi: 10.4049/jimmunol.0902425

27. Bertin J, DiStefano PS. The PYRIN domain: a novel motif found in apoptosis and inflammation proteins. Cell Death Differ. (2000) 7:12734. doi: $10.1038 /$ sj.cdd. 4400774

28. Yang SJ, Shao GF, Chen JL, Gong J. The NLRP3 inflammasome: an important driver of neuroinflammation in hemorrhagic stroke. Cell Mol Neurobiol. (2018) 38:595-603. doi: 10.1007/s10571-017-0526-9

29. Malhotra S, Rio J, Urcelay E, Nurtdinov R, Bustamante MF, Fernandez O, et al. NLRP3 inflammasome is associated with the response to IFN-beta in patients with multiple sclerosis. Brain. (2015) 138:644-52. doi: 10.1093/brain/awu388

30. de Souza EE, Hehnly H, Perez AM, Meirelles GV, Smetana JH, Doxsey S, et al. Human Nek7-interactor RGS2 is required for mitotic spindle organization. Cell Cycle. (2015) 14:656-67. doi: 10.4161/15384101.2014.994988

31. Kim S, Lee K, Rhee K. NEK7 is a centrosomal kinase critical for microtubule nucleation. Biochem Biophys Res Commun. (2007) 360:5662. doi: 10.1016/j.bbrc.2007.05.206

32. Kimura M, Okano Y. Identification and assignment of the human NIMArelated protein kinase 7 gene (NEK7) to human chromosome 1q31.3. Cytogenet Cell Genet. (2001) 94:33-8. doi: 10.1159/000048779

33. Feige E, Motro B. The related murine kinases, Nek6 and Nek7, display distinct patterns of expression. Mech Dev. (2002) 110:219-23. doi: 10.1016/S0925-4773(01)00573-1

34. Salla M, Butler MS, Pelingon R, Kaeslin G, Croker DE, Reid JC, et al. Synthesis, and biological evaluation of the major human metabolite of NLRP3 inflammasome inhibitor MCC950. Acs Med Chem Lett. (2016) 7:10348. doi: 10.1021/acsmedchemlett.6b00198

35. Xu KY, Wu CY, Tong S, Xiong P, Wang SH. The selective Nlrp3 inflammasome inhibitor Mcc950 attenuates lung ischemia-reperfusion injury. Biochem Biophys Res Commun. (2018) 503:3031-7. doi: 10.1016/j.bbrc.2018.08.089

36. Zhang Y, Lv X, Hu Z, Ye X, Zheng X, Ding Y, et al. Protection of Mcc 950 against high-glucose-induced human retinal endothelial cell dysfunction. Cell Death Dis. (2017) 8:e2941. doi: 10.1038/cddis.2017.308

37. Luo Y, Lu J, Ruan W, Guo X, Chen S. MCC950 attenuated early brain injury by suppressing NLRP3 inflammasome after experimental SAH in rats. Brain Res Bull. (2019) 146:320-6. doi: 10.1016/j.brainresbull.2019.01.027

38. Ren H, Kong Y, Liu Z, Zang D, Yang X, Wood K, et al. Selective NLRP3 (Pyrin Domain-Containing Protein 3) inflammasome inhibitor reduces brain injury after intracerebral hemorrhage. Stroke. (2018) 49:18492. doi: 10.1161/STROKEAHA.117.018904
39. Chen I, Mitchell P. Serum potassium and sodium levels after subarachnoid haemorrhage. Br J Neurosurg. (2016) 30:5549. doi: 10.1080/02688697.2016.1181151

40. Dreier JP, Woitzik J, Fabricius M, Bhatia R, Major S, Drenckhahn C, et al. Delayed ischaemic neurological deficits after subarachnoid haemorrhage are associated with clusters of spreading depolarizations. Brain. (2006) 129:322437. doi: 10.1093/brain/awl297

41. Gross CJ, Mishra R, Schneider KS, Medard G, Wettmarshausen J, Dittlein DC, et al. $\mathrm{K}(+)$ Efflux-independent NLRP3 inflammasome activation by small molecules targeting mitochondria. Immunity. (2016) 45:761773. doi: 10.1016/j.immuni.2016.08.010

42. Xiong XY, Liu L, Yang QW. Functions and mechanisms of microglia/macrophages in neuroinflammation and neurogenesis after stroke. Prog Neurobiol. (2016) 142:23-44. doi: 10.1016/j.pneurobio.2016.05.001

43. Schneider UC, Davids AM, Brandenburg S, Muller A, Elke A, Magrini S, et al. Microglia inflict delayed brain injury after subarachnoid hemorrhage. Acta Neuropathol. (2015) 130:215-31. doi: 10.1007/s00401-015-1440-1

44. Yan N, Liu Y, Liu S, Cao S, Wang F, Wang Z, et al. Fluoride-induced neuron apoptosis and expressions of inflammatory factors by activating microglia in rat brain. Mol Neurobiol. (2016) 53:4449-60. doi: 10.1007/s12035-015-9380-2

45. Hinojosa AJ, Deogracias R, Rico B. The microtubule regulator NEK7 coordinates the wiring of cortical parvalbumin interneurons. Cell Rep. (2018) 24:1231-42. doi: 10.1016/j.celrep.2018.06.115

46. Freixo F, Martinez Delgado P, Manso Y, Sanchez-Huertas C, Lacasa C, Soriano E, et al. NEK7 regulates dendrite morphogenesis in neurons via Eg5-dependent microtubule stabilization. Nat Commun. (2018) 9:2330. doi: 10.1038/s41467-018-04706-7

47. Valdinocci D, Grant GD, Dickson TC, Pountney DL. Epothilone D inhibits microglia-mediated spread of alpha-synuclein aggregates. Mol Cell Neurosci. (2018) 89:80-94. doi: 10.1016/j.mcn.2018.04.006

48. Misawa T, Takahama M, Kozaki T, Lee H, Zou J, Saitoh T, et al. Microtubuledriven spatial arrangement of mitochondria promotes activation of the NLRP3 inflammasome. Nat Immunol. (2013) 14:454-60. doi: 10.1038/ni.2550

49. Keaney J, Campbell M. The dynamic blood-brain barrier. FEBS J. (2015) 282:4067-79. doi: 10.1111/febs.13412

50. Yang F, Wang Z, Wei X, Han H, Meng X, Zhang Y, et al. NLRP3 deficiency ameliorates neurovascular damage in experimental ischemic stroke. J Cereb Blood Flow. (2014) 34:660-7. doi: 10.1038/jcbfm.2013.242

Conflict of Interest: The authors declare that the research was conducted in the absence of any commercial or financial relationships that could be construed as a potential conflict of interest.

Copyright (C) 2020 Li, Dong, Liu, Zou, Hao, Gao, Pan and Liang. This is an openaccess article distributed under the terms of the Creative Commons Attribution License (CC BY). The use, distribution or reproduction in other forums is permitted, provided the original author(s) and the copyright owner(s) are credited and that the original publication in this journal is cited, in accordance with accepted academic practice. No use, distribution or reproduction is permitted which does not comply with these terms. 